\title{
Zum Russischunterricht an drei Lernorten im Ruhrgebiet - eine empirische Studie
}

\author{
Katrin Bente Karl (Bochum)
}

Статья преАставцяет ознакомительный обзор студенческого проекта, целью которого явАяется выяснение ситуации с предметом «Русский язык как иностранный» в трех учебных заведениях. В рамках семинара магистратуры было разработано и проведено исследование, состоящее из интервью и опросников. Полученные таким образом Аанные, состоящие из ответов преподавателей и учащихся, явАяются темой Аанной статьи. При этом мы рассмотрим разные перспективы: сначала будут представлены результаты опроса учителей, касающиеся организации занятий. Затем будет Аана общая информация об учащихся, из которой вытекает количественное соотношение изучающих русский как иностранный и как язык семейного общения, а также причины и мотивация изучения русского языка. В последней части будет описана информация об уровне знаний русского языка учащихся и результаты (частично проведенных студентами) специальных исследований по вопросам оценки успеваемости, связи межАу мотивацией и результатами тестов, ассоциативного поведения и навыков чтения.

\section{Einleitung: Fragestellung und Zielsetzung}

Im Rahmen eines Hauptseminars an der Ruhr-Universität Bochum im Wintersemester 2015/16 mit dem Titel „Linguistische Aspekte des Russischen als Fremdsprache“ wurde unter meiner Leitung von den TeilnehmerInnen eine empirische Studie designt und an drei unterschiedlichen Lernorten des Russischen als 
Fremdsprache (RAF) durchgeführt. ${ }^{1}$ Ziel des Projektes war es, einen möglichst breiten Einblick in den aktuellen Stand des Russischunterrichts an diesen Lernorten zu bekommen. Dafür sollten möglichst viele Aspekte abgedeckt werden: die LehrerInnenperspektive (inkl. der Frage nach dem didaktischen Konzept und den eingesetzten Lehrmaterialien), die SchülerInnenperspektive (inkl. sprachbiographischer Daten und linguistischer Kompetenzen im Russischen) und die Frage nach der Art der Lehrinstitution. Mit diesem Aufbau konnte die von Targońska (2012) empfohlene Einbeziehung der drei Perspektiven (Lehrende, Lernende und Lehrwerke) erreicht werden. Des Weiteren kam in der theoretischen Vor- und Aufbereitung die sprachliche Ebene als weiterer Faktor hinzu. In diesem Zusammenhang erfolgte eine Auseinandersetzung mit Ähnlichkeiten und Unterschieden im Russischen und Deutschen und deren Auswirkungen auf den Erwerb des RAF. Als Untersuchungsinstrumente kamen das leitfadenorientierte Interview für Gespräche mit den Lehrpersonen, schriftliche Fragebögen zur Abfrage allgemeiner sprachbiographischer sowie linguistischer Daten der SchülerInnen und schließlich eine mündliche Aufnahme im Bereich der Phonetik zum Einsatz. Mit diesem Aufbau konnten umfangreiche Daten von insgesamt 36 Lernenden des Russischen und sechs Lehrpersonen ${ }^{2}$ an drei unterschiedlichen Lernorten - einem Gymnasium, einem privaten Weiterbildungskolleg und einer Universität - gewonnen werden. Ziel des vorliegenden Beitrags ist es, Einblicke in die erhobenen Daten zu liefern und exemplarische Fragestellungen und Ergebnisse herauszugreifen und darzustellen. Zu diesem Zweck soll als Erstes die Datenerhebung genauer beschrieben werden. In der daran anknüpfenden Auswertung der Daten sollen zunächst zentrale Ergebnisse aus den LehrerInneninterviews vorgestellt werden. Dem folgt eine Zusammenschau einiger relevanter Faktoren aus den (sprach-)biographischen Angaben der SchülerInnen. Im dritten Schritt soll ein Blick auf die gewonnenen Sprachdaten geworfen werden. Die durchgeführte Untersuchung hat dabei eine Fülle an Sprachdaten hervorgebracht, die

1 Mein Dank geht an alle TeilnehmerInnen dieses Seminars, ohne deren aktive Unterstützung diese Ergebnisse und auch diese Publikation nicht zu Stande gekommen wären

2 Ein sehr herzlicher Dank geht hier an alle Personen, die sich an der Umfrage beteiligt haben und mir und meinen Studierenden dankenswerterweise bereitwillig umfassende Einblicke in ihren Lehr- und Lernalltag gewährt haben.

Anka Bergmann, Olga Caspers \& Wolfgang Stadler (Hg.)

Didaktik der slawischen Sprachen - Beiträge zum 1. Arbeitskreis in Berlin (12.-14.9.2016)

(C) 2018 innsbruck university press, ISBN 978-3-903187-11-5, DOI 10.15203/3187-11-5 
im Rahmen dieses Beitrages nicht erschöpfend dargestellt und analysiert werden können. Um einen Eindruck davon zu vermitteln, welche linguistischen Fragen aus dem Seminar heraus von Studierenden selbst an das Material gestellt wurden, soll hier der Weg gewählt werden, exemplarische Arbeiten von Studierenden mit ihren zentralen Ergebnissen zu präsentieren. Ergänzend soll eine eigene Auswertung zur Vorlesekompetenz die linguistische Auswertung abrunden. Abschließend folgt ein zusammenfassender Ausblick.

\section{Datenerhebung}

Die Erhebung der Daten wurde im Dezember 2015 und Januar 2016 an insgesamt drei Lernorten durchgeführt: An einem staatlichen Gymnasium nahmen zwei Lernerklassen und Lehrpersonen des Russischen teil, dies waren insgesamt 28 SchülerInnen der zehnten und elften Stufe. Die neu einsetzende Fremdsprache Russisch wird an diesem Gymnasium ab der achten Klasse angeboten und nicht in weitere Gruppen (wie z. B. HerkunftssprecherInnen) unterteilt. Der Unterricht in der zehnten und elften Stufe findet zweimal wöchentlich (à 90 Minuten) statt und wird von unterschiedlichen Lehrmaterialien begleitet (s. u.).

Als zweiter Lernort kam ein privates Weiterbildungskolleg nach der Pädagogik Rudolf Steiners hinzu - hier nahmen neun SchülerInnen und deren Lehrkraft teil. Der Russischunterricht an diesem Weiterbildungskolleg schließt (mit Unterbrechung durch eine berufsqualifizierende Phase) an den Russischunterricht der im Regelfall zuvor besuchten Schule an. Dort wird Russisch ab der ersten Klasse als zweite Fremdsprache nach dem Prinzip der Waldorfschulen zunächst spielerisch und rhythmisch orientiert angeboten und bis zur zwölften Klasse fortgeführt. Nach Abschluss der zwölften Klasse führt der Besuch des Kollegs zum Abitur. In diesem Abschnitt ist Russisch als prüfungsrelevantes Fach (Grundkurs) freiwillig wählbar und wird stufenübergreifend und ebenfalls nicht in weitere Gruppen unterteilt angeboten.

Den dritten Lernort bildet die Universität ab, an der sechs Studierende der Slawistik und ihre Lehrkraft befragt wurden. Sie befanden sich zum Zeitpunkt der Befragung im dritten Semester und besuchten den Aufbaukurs I des Russi-

Anka Bergmann, Olga Caspers \& Wolfgang Stadler (Hg.)

Didaktik der slawischen Sprachen - Beiträge zum 1. Arbeitskreis in Berlin (12.-14.9.2016)

(C) 2018 innsbruck university press, ISBN 978-3-903187-11-5, DOI 10.15203/3187-11-5 
schen mit vier Semesterwochenstunden Unterricht (dies setzt den erfolgreichen Besuch der Grundkurse aus den ersten zwei Semestern bzw. eine entsprechende Befreiung durch einen Einstufungstest voraus). Hinsichtlich des Fremdspracherwerbs (sowohl bzgl. der Dauer als auch der Art) liegen damit in diesen drei Lernorten unterschiedliche Bedingungen vor, die bei der Auswertung der erhobenen Daten beachtet werden müssen. Mit Hinblick auf das zu erwartende Sprachniveau ist ebenfalls von Unterschieden bzw. einer starken Heterogenität auszugehen, die bei diesem Untersuchungsdesign bedacht werden musste. Nach Rückversicherung bei den Lehrenden ist von einer Sprachkompetenz, die zumindest dem GeR-Niveau A1/A2 entspricht, auszugehen, in einigen Fällen (besonders bei den HerkunftssprecherInnen) ist mit weitaus höheren Kompetenzen zu rechnen.

Die Befragung bestand aus zwei Teilen (s. u.), an denen insgesamt 36 Lernende teilnahmen. Von Seiten der Lehrkräfte wurden sechs Personen befragt: die vier Lehrpersonen der befragten Klassen sowie zwei weitere Lehrer aus zwei Gymnasien in Nordrhein-Westfalen. Da von diesen beiden Lehrkräften jedoch keine Klassenbefragung erfolgen konnte, werden die Daten aus diesen Gesprächen nur z. T. kontrastiv angeführt.

Die Entwicklung der Befragung fand im Seminar statt und wurde hauptsächlich von den Studierenden erarbeitet, im Seminar diskutiert und dann umgesetzt. Dort wurde auch eine Zweiteilung der Befragung entschieden, die sich in einen allgemeinen Fragebogen und eine linguistische Befragung unterteilte. Der erste Fragebogen enthielt offene und geschlossene Fragen, die teils dichotom, teils nach Antwortkategorien gegliedert waren und die folgenden Themenbereiche abdeckten:

- Sprachbiographischer Hintergrund mit Fragen zum Spracherwerb (Erst-I Zweitsprache/n, Art und Dauer des Erwerbs etc.);

- Motivationen des Russischlernens (Gründe und aktuelle Zufriedenheit);

- Selbsteinschätzung der Russischkenntnisse (6-teilige Skala, aufgegliedert nach den vier Sprachfertigkeiten);

- Kenntnisstand/Behandlung im Unterricht von Grammatik und Wortschatz (Abfrage der Behandlung der Kasus und der Thematisierung von Lerntechniken); 
- Eigenständige Auseinandersetzung mit und Verwendung des Russischen über den Unterricht hinaus.

Dieser erste Teil der Befragung (der insgesamt sechs Seiten umfasste und im Weiteren als „allgemeiner Fragebogen“ bezeichnet wird) wurde über die Lehrpersonen im Unterricht an die LernerInnen verteilt und im Laufe einer Unterrichtsstunde ausgefüllt und eingesammelt. Dies geschah zwei Wochen vor dem zweiten Teil der Untersuchung. Um eine Zuordnung des ersten und zweiten Teils und zugleich die Anonymität zu gewährleisten, wurde von jedem/jeder ProbandIn ein eindeutiger, verschlüsselter Code erstellt und auf den Fragebögen (und der mündlichen Befragung) notiert. ${ }^{3}$

Der zweite Teil der Datenerhebung widmete sich der Feststellung konkreter linguistischer Kompetenzen im Russischen. Die dafür verwendeten Testformate wurden von den Studierenden in thematischen Gruppen erarbeitet und an den drei Lernorten eigenständig durchgeführt. Die abgedeckten Kompetenzbereiche waren Wortschatz (inkl. Lernstrategien und Assoziationen), Grammatik, Wortbildung und Phonetik. Die ersten drei Gebiete wurden (in der genannten Reihenfolge) über einen schriftlichen Fragebogen abgeprüft, wobei sich der Aufbau der einzelnen Bereiche jeweils ähnelte. Zu Beginn standen allgemeine Fragen, wie z. B. jene nach im Unterricht behandelten Aspekten („Welche Fälle wurden bislang im Unterricht behandelt? - Kreuze an: Nominativ Singular, Genitiv Singular ..."), dem folgten Aufgaben unterschiedlicher Art mit steigendem Schwierigkeitsgrad. Eine Orientierung bei der Erstellung der Aufgaben lieferte dabei die Fachdidaktik Russisch (Bergmann 2014), eine produktive Wortbildungsaufgabe wurde aus Stadler (2014: 301 aus eben diesem Band) übernommen. Dabei wurden, je nach Themengebiet, unterschiedliche Testformate unter Rückgriff auf vorhandene Untersuchungen verwendet. Für den Bereich der Grammatik (für den u. a. eine Orientierung an Hartenstein [2000] stattfand) wurde in einem ersten Schritt explizites Kasuswissen abgefragt (durch Aufforderung, vorgegebene

3 Aus Platzgründen muss hier leider auf eine ausführliche Darstellung der einzelnen Bestandteile der Fragebögen sowie der Generierung des Codes verzichtet werden. Bei Interesse sind diese Informationen gern einsehbar (bitte wenden Sie sich per E-Mail an Katrin.Karl@rub.de).

Anka Bergmann, Olga Caspers \& Wolfgang Stadler (Hg.)

Didaktik der slawischen Sprachen - Beiträge zum 1. Arbeitskreis in Berlin (12.-14.9.2016)

(C) 2018 innsbruck university press, ISBN 978-3-903187-11-5, DOI 10.15203/3187-11-5 
Wörter in einen bestimmten Kasus zu setzen), dem folgte die Aufgabe, vorgegebene Wörter im Satzkontext in den korrekten (nicht vorgegebenen Kasus) zu setzen und die Wahl zu begründen. Im Anschluss daran sollten kasusspezifische Fragen mithilfe eines selbst formulierten Satzes beantwortet werden (z. B. Čem Vy sejčas pišete?). Für die Wortbildung erfolgte eine Orientierung an Trušina (1974). Im Mittelpunkt stand die Frage, ob Wortbildungsprodukte zu zusammengehörigen Gruppen angeordnet oder passende Suffixe ihren Basen zugeordnet werden konnten. Ein Lückentext fragte abschließend produktive Kenntnisse ab. Für den Assoziationstest wurde auf die Untersuchung von Anstatt (2008) zurückgegriffen und diese in verkürzter Form übernommen.

Mithilfe dieser Aufgaben wurden unterschiedliche Kompetenzbereiche abgedeckt und jeweils unterschiedliche Fähigkeiten der LernerInnen abgefragt. Eine wichtige Unterteilung war dabei jene in implizites vs. explizites Wissen (vgl. Raupach 2002, Röhr-Sendlmeier 2012). Dies war in der Seminardiskussion zum Gegenstand geworden, da im Vorfeld mit einer sehr heterogenen Lernergruppe gerechnet wurde, die durch mögliche Unterschiede im Spracherwerb unterschiedliche Arten von sprachlichem Wissen im Russischen aufweisen. So war eine Hypothese, dass HerkunftssprecherInnen des Russischen über stärker ausgeprägtes implizites Wissen verfügen und damit alle Lückentexte problemlos ausfüllen können sollten, während FremdsprachenlernerInnen evtl. über mehr explizites Wissen verfügen und damit Fragen zur Begründung von z. B. Kasusverwendungen beantworten, aber nicht so problemlos deren korrekte Satzverwendung umsetzen können. Die Anordnung der Aufgaben innerhalb eines Kompetenzbereichs erfolgte dabei bewusst von sehr leicht hin zu komplexer, um den zu erwartenden unterschiedlichen Sprachniveaus gerecht zu werden. So war die Hoffnung, dass alle etwas ihrem Niveau Entsprechendes auffinden können. Ein weiteres Problem bei der Aufgabenentwicklung stellte die Implementierung unbekannter Testformate dar. So musste davon ausgegangen werden, dass bspw. Assoziationstests bislang nicht Gegenstand im Fremdsprachenunterricht waren oder auch Aufgaben zur expliziten Wortbildung selten im Fokus stehen. Da diese Themen jedoch im Seminarinteresse lagen, entschied man sich dazu, passende Formate für die einzelnen Kompetenzbereiche zu suchen und sich nicht daran zu orientieren, was den SchülerInnen aus dem Unterricht bekannt ist. Auch dies hat

Anka Bergmann, Olga Caspers \& Wolfgang Stadler (Hg.)

Didaktik der slawischen Sprachen - Beiträge zum 1. Arbeitskreis in Berlin (12.-14.9.2016)

(C) 2018 innsbruck university press, ISBN 978-3-903187-11-5, DOI 10.15203/3187-11-5 
zwangsläufig Auswirkungen auf die zu erwartenden Ergebnisse und muss bei der Interpretation berücksichtigt werden (s. u.).

Die Durchführung geschah an allen drei Lernorten im Unterricht (hierfür wurden 90 Minuten des Fachunterrichts zur Verfügung gestellt) unter der Leitung der Studierenden. Nach der Bearbeitung der schriftlichen Aufgaben (im Umfang von zehn Seiten) wurden die LernerInnen einzeln zur mündlichen Befragung gebeten. Diese fand zum Thema der Phonetik in einem gesonderten Raum statt. In diesem Teil wurden die LernerInnen zunächst von zwei Studierenden zu spezifischen Schwierigkeiten im Bereich der Aussprache des Russischen und ihrer Thematisierung (bzw. auch ihres Wunsches nach expliziter Behandlung) im Unterricht befragt und dann gebeten, einen kurzen Text (auf Stufe A1 - ohne Betonungszeichen) sowie einzelne Wörter (auch wiederum nach Schwierigkeitsgrad in Aussprache und Orthographie gestuft) laut vorzulesen.

Eine weitere Studierendengruppe beschäftigte sich mit der LehrerInnenbefragung. Hierfür wählten sie als Instrument das fragebogengeleitete Interview und erarbeiteten Fragen zum (sprach-)biographischen Hintergrund der Lehrperson, der methodisch-didaktischen Ausrichtung ihres Unterrichts (inkl. der Frage nach dem Einsatz des Lehrmaterials) sowie konkreter Fragen zu den einzelnen behandelten Kompetenzbereichen (s. o.: Grammatik, Wortschatz, Wortbildung und Phonetik) und deren didaktischer Aufbereitung sowie der Einschätzung des Lernstands der jeweiligen Klasse. Allgemeine Fragen zur Zusammensetzung der Klasse (z. B. HerkunftssprecherInnen bzw. Besonderheiten durch andere mehrsprachige Konstellationen) rundeten die Befragung ab. Diese fand mündlich mit jeweils einer (bzw. in einem Fall zwei) Lehrperson(en) aller befragten Klassen statt.

Durch das Design der Untersuchung und deren Durchführung an drei sehr unterschiedlichen Lernorten sollte bewusst die Breite und Vielfalt des Russischerwerbs in den Vordergrund gerückt werden. Wie aus der obigen Schilderung ersichtlich, hatte das Seminar im Vorfeld dabei mit Schwierigkeiten unterschiedlicher und z. T. auch bewusst hervorgerufener Art zu tun. Diese äußern sich zusammenfassend v. a. in der zu erwartenden (und beabsichtigten) Heterogenität der Sprachvermittlung zwischen den drei Lernorten, in der zu erwartenden Heterogenität in der SchülerInnenschaft hinsichtlich ihres Spracherwerbs (HerkunftssprecherInnen des Russischen, einer anderen Sprache als Deutsch und Russisch

Anka Bergmann, Olga Caspers \& Wolfgang Stadler (Hg.)

Didaktik der slawischen Sprachen - Beiträge zum 1. Arbeitskreis in Berlin (12.-14.9.2016)

(C) 2018 innsbruck university press, ISBN 978-3-903187-11-5, DOI 10.15203/3187-11-5 
und des Deutschen) und im Einsatz von den SchülerInnen unbekannten Testformaten. Diese Aspekte wurden im Seminar thematisiert und bestmöglich im Diskurs aufgegriffen.

Die Studie ist als eine breitangelegte explorative Untersuchung zu verstehen, die nicht den Anspruch erhebt, einzelnen Fragen bis ins kleinste Detail auf den Grund zu gehen, sondern vielmehr einen Eindruck von den unterschiedlichen Arten des Russischerwerbs, der Russischvermittlung und deren eventuellen Auswirkungen auf bestimmte Russischkompetenzen bei den Lernenden vermitteln soll.

\section{Auswertung der Daten: Einblicke in exemplarische Fragestellungen und deren Ergebnisse}

Wie aus der Beschreibung der Datenerhebung ersichtlich, liegt eine Fülle an gesammeltem Material vor. In die Auswertung der Daten soll an dieser Stelle ein exemplarischer Einblick vorgenommen werden, detailliertere Ergebnisse bzw. notwendige vertiefende Studien können in diesem Rahmen nicht geleistet werden, sollen aber explizit angeregt werden.

Im Folgenden werden zunächst die qualitativ ausgewerteten Ergebnisse der LehrerInnendaten dargestellt, dem schließt sich ein Überblick über die Angaben aus dem allgemeinen Teil des Fragebogens der LernerInnen an. In einem dritten Schritt sollen drei exemplarische Studienarbeiten herangezogen werden, um darzustellen, welche (linguistischen) Fragestellungen aus dem Seminar von den TeilnehmerInnen bearbeitet wurden und zu welchen Ergebnissen diese führten. Dieser Teil wird durch eine weitere Untersuchung der Autorin zur Vorleseperformanz von 18 LernerInnen abgerundet.

\subsection{LehrerInnendaten}

Die vier Lehrkräfte, deren SchülerInnen in die Untersuchung eingeflossen sind, zeichnen sich alle durch die Gemeinsamkeit aus, dass sie nicht in Deutschland geboren sind, Russisch als ihre Erstsprache (und Deutsch als L2) erworben haben und keine weitere Fremdsprache unterrichten. Aufgrund ihrer drei- bis zehnjähri-

Anka Bergmann, Olga Caspers \& Wolfgang Stadler (Hg.)

Didaktik der slawischen Sprachen - Beiträge zum 1. Arbeitskreis in Berlin (12.-14.9.2016)

(C) 2018 innsbruck university press, ISBN 978-3-903187-11-5, DOI 10.15203/3187-11-5 
gen Berufserfahrung sind sie einer jüngeren LehrerInnengeneration zuzuordnen. Diese Zuordnung fällt v. a. in Kontrast zu den zwei zusätzlich befragten Lehrpersonen auf, deren Erstsprache Deutsch ist, die jeweils eine weitere Fremdsprache unterrichten und mit 35-jähriger Berufserfahrung einer älteren LehrerInnengeneration angehören. Diesen Generationsunterschied kann man auch aus dem Einsatz der Lehrmaterialien und der Zufriedenheit der Lehrkräfte ablesen. Während die beiden Gymnasiallehrkräfte der älteren Generation mit jeweils einem Lehrwerk arbeiten (Конечно bzw. Аиалог), damit zufrieden sind und auf eine explizite Grammatikvermittlung und schriftliche Orientierung setzen, arbeiten die beiden jüngeren Gymnasiallehrer mit Materialien aus unterschiedlichen Lehrwerken (Привет, Окно, Конечно, Аиалог), sind dabei mit keinem wirklich zufrieden, versuchen Grammatik kontextuell einzuführen und sind eher auf mündliche Produktion orientiert. Sie setzen alternative Lehr- und Lernmethoden ein (wie z. B. die Ampelmethode in der Wortschatzvermittlung und Lerngruppen über WhatsApp) und haben als oberste Maxime, Spaß am Russischlernen zu vermitteln. Alle Gymnasiallehrkräfte führen als größtes Problem die Zeitknappheit an, der viele wichtige Bereiche des Fremdsprachenunterrichts zum Opfer fallen würden.

Die Schilderung des Lernalltags am Weiterbildungskolleg grenzt sich davon in einigen Punkten ab. Betont wird hier die Wichtigkeit der mündlichen und rhythmischen Seite im Spracherwerb (v. a. in den unteren Klassen), der bereits in der ersten Klasse einsetzt, was eine lange Kontaktzeit mit der Sprache bedeutet. Die Lehrkraft verwendet kein Lehrwerk, sondern stellt sich ihre Materialien aus unterschiedlichen Medien selbst zusammen. Grammatik wird häufig induktiv erschlossen, aber im Anschluss explizit, auch kontrastiv, besprochen. Besonders betont wurde hier auch die Möglichkeit, verschiedene Lernstrategien (wie z. B. im Bereich der Wortschatzarbeit) thematisieren zu können. Dieser Ansatz der Sprachvermittlung wird explizit auf die im Laufe des gesamten Schullebens zur Verfügung stehende umfangreiche Unterrichtszeit und die daraus resultierenden Freiräume zurückgeführt.

Die Ausrichtung des Unterrichts an der Universität ist auf andere Aspekte gelenkt. Hier steht im Anfängerunterricht v. a. eine umfangreiche und explizite Grammatikvermittlung sowie Textarbeit im Vordergrund. Mit steigender Progression kommt die mündliche Produktion hinzu. In den Anfängerkursen dient

Anka Bergmann, Olga Caspers \& Wolfgang Stadler (Hg.)

Didaktik der slawischen Sprachen - Beiträge zum 1. Arbeitskreis in Berlin (12.-14.9.2016)

(C) 2018 innsbruck university press, ISBN 978-3-903187-11-5, DOI 10.15203/3187-11-5 
ein aus unterschiedlichen Lehrwerken zusammengestelltes Skript als Lerngrundlage. Mangel an Zeit für die Behandlung von vertiefenden Fragen bzw. für die Einübung der vermittelten Regeln wird hier von der Lehrkraft - ebenso wie an den Gymnasien - als größtes Problem identifiziert.

Auf die relevanten Ergebnisse der LehrerInnenbefragung hinsichtlich der Einschätzung des Kenntnisstandes ihrer Klassen wird an den entsprechenden Stellen weiter unten eingegangen.

\subsection{Allgemeine LernerInnendaten}

In die Auswertung der allgemeinen LernerInnendaten gehen die Angaben von insgesamt 36 ProbandInnen ein. Das Alter der befragten Personen lag zwischen 17 und 23 Jahren (nach den unterschiedlichen Lernorten aufgegliedert zeigt sich ein jeweils sehr homogenes Bild: Gymnasium 17-18 Jahre, Kolleg 19-20 Jahre und Universität 21-23 Jahre), es beteiligten sich 11 Männer und 25 Frauen. Auf die Frage nach ihrer Erstsprache gaben 22 Personen nur Deutsch an (61\%), bei sieben (19\%) hingegen ist Russisch die Erstsprache (=L1) und bei weiteren sieben lässt sich von einem bilingualen Spracherwerb sprechen: Drei gaben Polnisch und Deutsch als parallel erworbene Sprachen an (also zwei L1), weitere vier Russisch und Deutsch (mit jeweiliger aktueller Dominanz des Deutschen). Fasst man die Personen mit herkunftssprachlichem russischem Hintergrund (jedweder Art) auf dieser Basis zusammen, so entspricht diese Gruppe 30,5\% aller ProbandInnen.

Bei der (mehrfach möglichen) Benennung der Motive für die Wahl des Russischen als Unterrichtsfach kann man eine klare Unterteilung in HerkunftssprecherInnen und FremdsprachenlernerInnen erkennen: Für erstere steht der Faktor der Vertiefung der Kenntnisse in der Muttersprache an erster Stelle (dies gaben zehn von elf HerkunftssprecherInnen an), während ein Großteil der FremdsprachenlernerInnen ein Interesse an der Sprache oder Schrift bzw. Relevanz für die (berufliche) Zukunft angab. Eine Besonderheit bei der Angabe der Motivation stellt die Gruppe der ProbandInnen aus dem Weiterbildungskolleg dar, die alle ihre Vorkenntnisse als einen Faktor angaben. Daneben wurde auch die (geringe) Kursgröße und damit einhergehend das bessere Betreuungsverhältnis als Grund für die Sprachenwahl genannt.

Anka Bergmann, Olga Caspers \& Wolfgang Stadler (Hg.)

Didaktik der slawischen Sprachen - Beiträge zum 1. Arbeitskreis in Berlin (12.-14.9.2016)

(C) 2018 innsbruck university press, ISBN 978-3-903187-11-5, DOI 10.15203/3187-11-5 
Auch in Bezug auf die Frage nach der Verwendung des Russischen außerhalb der Schule gibt es einen klaren Unterschied zwischen den HerkunftssprecherInnen und den FremdsprachenlernerInnen. Während letztere bis auf zwei Ausnahmen (hier liegt ein jeweils russischsprachiger Partner vor) nur in der Schule Russisch verwenden, gaben die HerkunftssprecherInnen (allerdings in unterschiedlichem Ausmaß) an, Russisch auch in der Familie und mit Freunden zu sprechen - wobei alle ProbandInnen ausnahmslos ausschließlich auf die mündliche Produktion und Rezeption verwiesen, niemand bezog sich auf die schriftliche Ebene.

Die Frage, in welchem Maße die LernerInnen Spaß am Russischlernen haben, beantworteten die ProbandInnen auf einer vierstufigen Likert-Skala („meistens“, „oft“, „selten“ und „nie“). Die meisten Antworten entfielen dabei auf die höchste Kategorie (42\%); zusammengenommen mit der zweiten (=25\%) lässt sich festhalten, dass $67 \%$ der Befragten Spaß am Russischlernen haben, während lediglich 33\% selten oder nie Spaß daran empfinden. Betrachtet man die Ergebnisse antwortnormiert, so lässt sich interessanterweise ein größerer Anteil bei den Prozentzahlen an HerkunftssprecherInnen mit angegebenem niedrigen Spaß am Russischlernen feststellen (im Umkehrschluss lässt sich folgern, dass FremdsprachenlernerInnen mehr Spaß am Russischlernen haben).

Im Rahmen des allgemeinen Fragebogens wurde ebenso der Grad der Beherrschung der russischen Sprache in Selbsteinschätzung (unterteilt nach den vier Sprachfertigkeiten) abgefragt. Auf die Ergebnisse und die Aussagekraft dieser Einschätzung wird im nächsten Abschnitt eingegangen.

\subsection{Fragestellungen und Ergebnisse aus exemplarischen studentischen Arbeiten}

In diesem Abschnitt sollen exemplarisch die Fragestellungen und Ergebnisse aus drei studentischen Arbeiten, die aus diesem Projekt heraus entwickelt wurden, vorgestellt werden. ${ }^{4}$ Die Seminararbeit von Bobb (2016) greift die Frage nach

4 Es sind im Rahmen des Seminars noch viele weitere, sehr interessante Arbeiten entstanden, aus Platzgründen fiel die Auswahl auf lediglich drei, die möglichst unterschiedliche Themen und Herangehensweisen abdecken. 
dem Zusammenhang von unterschiedlichen Möglichkeiten der Leistungsbeurteilung auf, Kandt (2016) konzentriert sich auf die Grammatikperformanz und deren Beeinflussung durch die Lernmotivation sowie den Spaß am Grammatiklernen und die Bachelorarbeit von Schlotthauer (2016) widmet sich umfangreich den Besonderheiten der Wortassoziationen von FremdsprachenlernerInnen des Russischen.

Bobb (2016) geht in ihrer Arbeit einer doppelten Fragestellung nach, indem sie untersuchte, inwiefern in einem ersten Schritt die LehrerInneneinschätzung der Leistung aller SchülerInnen der eigenen Klasse (im Klassenmittel) mit der Selbsteinschätzung dieser SchülerInnen aus dem Fragebogen übereinstimmt. In einem zweiten Schritt legte Bobb die Daten aus dem speziellen Fragebogenteil zur Grammatik und Wortbildung zu Grunde, wertete diese in Hinblick auf die tatsächlich geleistete Performanz aus und glich diese Leistung mit den Einschätzungen aus dem ersten Teil ab. Um die unterschiedlichen Bewertungsarten in eine vergleichbare Skala zu setzen, übertrug Bobb die jeweiligen Bewertungen in das deutsche Schulnotensystem (1-6, unter Differenzierung mithilfe von + und -), welches auch die LehrerInnen bei der Klasseneinschätzung verwendeten. In ihre Untersuchung flossen die Daten von 21 SchülerInnen aus dem Gymnasium und neun aus dem Weiterbildungskolleg mit ihren befragten drei Lehrkräften ein, die Ergebnisse unterzog sie einer statistischen Überprüfung. Interessant an dieser Fragestellung ist der direkte Vergleich von drei sehr unterschiedlichen, durchaus kontrovers angesehenen Leistungsbeurteilungsmethoden. In der Einschätzung der LehrerInnen werden jene als BewertungsexpertInnen angesehen, die ihre Bewertungskompetenz durch die Beobachtung ihrer SchülerInnen während eines längeren Zeitraums, durch den Abgleich mit Lehrkonzepten und -zielen sowie im Vergleich mit anderen Klassen erwerben, auch wenn sie dabei von emotionalen Urteilen beeinflusst sind. In der konkreten Situation der Befragung eines/ einer LehrersIn nach dem Kompetenzniveau der eigenen SchülerInnen kann es etwa zu einer selbstwertdienlichen Überschätzung kommen. Die LehrerInnen beurteilten im mündlichen Gespräch das durchschnittliche Leistungsniveau ihrer gesamten Klasse. Das Messinstrument der Selbsteinschätzung, die die SchülerInnen in schriftlicher Form abgaben, gilt zwar nicht als unumstrittene Methode, wird jedoch als bekanntes Messinstrument schon sehr lang verwendet und ist

Anka Bergmann, Olga Caspers \& Wolfgang Stadler (Hg.)

Didaktik der slawischen Sprachen - Beiträge zum 1. Arbeitskreis in Berlin (12.-14.9.2016)

(C) 2018 innsbruck university press, ISBN 978-3-903187-11-5, DOI 10.15203/3187-11-5 
zudem z. B. in einem Bericht der HISBUS 5 (Peschel, Senger \& Willige 2006) aufgearbeitet worden. Dort stellten die Autoren eine gute Übereinstimmung von Selbsteinschätzung und Testergebnissen für Englisch als Fremdsprache unter Studierenden fest (vgl. ebd.: 35). Je nach Charakter der befragten Person ist eine Unter- oder auch Überschätzung der Fähigkeiten möglich, selten jedoch in stark ausgeprägtem Maße.

Mit den sprachlichen Testergebnissen aus dem Fragebogen liegt eine dritte, objektivere - dabei allerdings nicht validierte - Grundlage vor, die ein Schlaglicht auf die Sprachperformanzen wirft. Die Ergebnisse zur ersten Fragestellung ergaben insgesamt eine grundsätzliche Übereinstimmung zwischen Fremd- und Selbsteinschätzung: So bewerteten die Lehrpersonen aus dem Gymnasium ihre Klassen im gesamten Klassendurchschnitt im Bereich einer 3+ (dabei mit besseren Kenntnissen in der Grammatik als in der Wortbildung), während die Lehrperson aus dem Weiterbildungskolleg ihre im Bereich einer 3 einstufte (mit ebenfalls besserer Einschätzung der Grammatik als der Wortbildung). Die Mittelwerte der Selbsteinschätzung ergaben für das Gymnasium eine 3 (bei stärkerer Ausnutzung der Bewertungsskala durch Verwendung von 2, 3 und 4), für das Weiterbildungskolleg ebenso (bei zugleich geringerer Ausnutzung der Skala wurde von keiner Person die 2 als Bewertungsmaßstab herangezogen, nur 3 und 4, woraus ein leicht niedrigerer Sprachstand geschlussfolgert werden kann). Die Ergebnisse aus der Auswertung des sprachlichen Tests waren in Bezug auf die Unterschiede zwischen den beiden Lernorten deckungsgleich: Das Gymnasium schnitt in Wortbildung und Grammatik jeweils besser ab als das Weiterbildungskolleg - dies ebenfalls passend um weniger als eine Schulnote. Dabei kam es jedoch zu zwei Abweichungen gegenüber der Einschätzung. Zum einen ergab die Testleistung eine schlechtere Note als die Einschätzung aus Teil I (für das Gymnasium zwischen 4+ und 4-, für das Weiterbildungskolleg zwischen 4- und 5), zum anderen fiel der Teil zur Wortbildung besser aus als jener zur Grammatik ${ }^{6}$. Dies

5 https://tinyurl.com/y7pgal6z [02.12.2017].

6 Dafür lassen sich sicherlich viele Gründe finden, die einerseits in der Art der Methode (s. o.) liegen und andererseits in der Art des Testformats, das für die Mehrzahl der SchülerInnen eine neue Testweise darstellte und möglicherweise zu Verunsicherung führte. Für die Diskrepanz hinsichtlich der Leistungen in der Wortbildung erscheint es notwendig darauf hinzuweisen, dass die Wortbildung bei keiner der Lehrpersonen

Anka Bergmann, Olga Caspers \& Wolfgang Stadler (Hg.)

Didaktik der slawischen Sprachen - Beiträge zum 1. Arbeitskreis in Berlin (12.-14.9.2016)

(C) 2018 innsbruck university press, ISBN 978-3-903187-11-5, DOI 10.15203/3187-11-5 
schmälert jedoch nicht den allgemeinen Eindruck der gut übereinstimmenden Ergebnisse aus den unterschiedlichen Möglichkeiten der Leistungsbeurteilung.

Eine zweite studentische Arbeit befasste sich mit der Frage, ob die getestete Grammatikperformanz mit der Lernmotivation an sich und dem abgefragten Spaß am Grammatiklernen in Zusammenhang steht. Dafür wertete Kandt (2016) die Daten von insgesamt zwölf ProbandInnen mit Deutsch als einziger L1 aus, die sich in der Angabe unterschieden, wie viel Spaß am Russischlernen allgemein und am Grammatiklernen im Speziellen sie haben (dies extrahierte sie aus den Daten des allgemeinen Fragebogens). Die Motivationsgrade glich sie mit den Ergebnissen aus dem Grammatikteil (des linguistischen Fragebogens) ab. In allen bis auf einen Fall (hier liegt vermutlich ein unerkannter Fall von russisch-deutscher Zweisprachigkeit vor, da bei der Person die Testergebnisse sehr gut waren und zugleich relevante Angaben aus dem soziolinguistischen Teil fehlten) steigt mit der Motivation und dem Grad an Spaß am Grammatiklernen die Grammatikperformanz im Test.

Eine letzte in diesem Kontext aufgegriffene Arbeit untersucht, inwiefern Assoziationen auf ausgewählte Wörter bei FremdsprachenlernerInnen des Russischen spezifische Charakteristika aufweisen. Schlotthauers Arbeit (2016) baut auf einem Artikel von Anstatt (2008) auf, die im freien Assoziationstest eine Bevorzugung von syntagmatischen Assoziationen im Russischen und Kontrastassoziationen im Deutschen nachweist und auf unterschiedliche Faktoren zurückführt. Der in den linguistischen Fragebogen dieser Untersuchung aufgenommene freie Assoziationstest greift eine Auswahl an kontrastfähigen Lexemen aus Anstatts Artikel heraus (wie z. B. belyj, mal'cik oder spat'). Schlotthauer analysierte die so gesammelten Assoziationen von insgesamt 20 ProbandInnen mit RAF (aus Gründen der Fragestellung wurden alle ProbandInnen, die Russisch im natürlichen Spracherwerb erworben haben, aus dieser Untersuchung ausgeschlossen) auf diese 28 Stimuli (Gesamtsumme 560) und betrachtete dabei im Besonderen die Art der Assoziation (syntagmatische Assoziationen vs. Kontrast-

\footnotetext{
im Unterricht aktiv behandelt wurde und daraus möglicherweise die schlechtere Einschätzung des Kenntnisstandes der SchülerInnen resultiert. Aus oben geschilderten Gründen wurde die Wortbildung jedoch bewusst in die Untersuchung integriert. Sie hat dabei auch eine Vielzahl an interessanten Ergebnissen gezeigt, die lediglich vor dem Hintergrund der unbekannten Testformate vorsichtiger behandelt werden müssen.
}

Anka Bergmann, Olga Caspers \& Wolfgang Stadler (Hg.)

Didaktik der slawischen Sprachen - Beiträge zum 1. Arbeitskreis in Berlin (12.-14.9.2016)

(C) 2018 innsbruck university press, ISBN 978-3-903187-11-5, DOI 10.15203/3187-11-5 
assoziationen), die Rolle der Übersetzung und der Lautlichkeit. Aus den Ergebnissen wird zunächst deutlich, dass Reaktionen fast ausschließlich auf Deutsch erfolgten und $17 \%$ davon als reine Übersetzung klassifiziert werden können. Daneben lässt sich ein hoher Anteil fehlender Assoziationen $(34 \%)^{7}$ feststellen. Unter den restlichen Assoziationen treten in etwa gleichen Anteilen individuelle Assoziationen auf (14\%, wie z. B. die Reaktion auf golova „Gedicht aus der ersten Klasse“), Klangassoziationen (16\%, z. B. auf bolezn’ „Bolognese“, oder auf radost' "Grillrost") und syntagmatische Assoziationen (18\%, z. B. auf devočka: „schön“ oder „jung"), wohingegen die Kontrastassoziationen nur mit 1\% vertreten waren (was im Kontrast zur bevorzugten Assoziationsart im Deutschen [vgl. dazu Anstatt 2008] steht). Diese Ergebnisse lassen vermuten, dass der Zugriff bei FremdsprachenlernerInnen auf russische Lexeme über das Deutsche stattfindet, was sich in der Übersetzung und der großen Bedeutsamkeit der lautlichen Seite zeigt. Diese dient, im Sinne von mnemotechnischen Hilfen (,Eselsbrücken') und einer Verknüpfung mit deutschen Formen, der Einprägung der Form. Die semantische Seite steht dabei nicht im Vordergrund, beim Abrufen ist eben jene lautliche Assoziation die stärkere. Dies zeigte bereits eine ältere Studie von Henning (1973), in der gezeigt wurde, dass FremdsprachenlernerInnen anfangs beim Vokabellernen klangliche Ähnlichkeiten zu anderen Sprachen suchen, während erst Fortgeschrittene in höherem Maße die Semantik berücksichtigen. Die Vormachtstellung der syntagmatischen Assoziationen zeigt sich, nach der bis heute vielfach zitierten Studie von Brown \& Berko (1960), auch im Spracherwerb von Kindern (hier des Englischen), die sich hin zu einer Bevorzugung von paradigmatischen Assoziationen (zu denen die Kontrastassoziationen auch zählen) im abgeschlossenen Spracherwerb entwickelt. Beide Ergebnisse sprechen dafür, dass aus den Ergebnissen des Assoziationstestes ein erst geringer Ausbau des Wortschatzes im Russischen hervorgeht.

7 Dies lässt sich wiederum aus der Befragungssituation verdeutlichen: Auch dieses Befragungsinstrument war sicher unbekannt und führte zu Verunsicherung und entsprechendem Nichtausfüllen; zudem ließe sich die Leerquote auch durch die Menge an Fragen und durch den entsprechenden Zeitdruck erklären.

Anka Bergmann, Olga Caspers \& Wolfgang Stadler (Hg.)

Didaktik der slawischen Sprachen - Beiträge zum 1. Arbeitskreis in Berlin (12.-14.9.2016)

(C) 2018 innsbruck university press, ISBN 978-3-903187-11-5, DOI 10.15203/3187-11-5 


\subsection{Einzelstudie: Vorleseperformanz}

Ergänzend zu den oben dargestellten exemplarischen Fragestellungen, die mithilfe des gesammelten Materials bearbeitet wurden und zu ersten Ergebnissen führten, soll hier, stellvertretend für den Themenbereich der Phonetik, ein Blick auf die Vorleseperformanz ausgewählter ProbandInnen geworfen werden. Dabei soll betrachtet werden, ob es zu Unterschieden kommt, die ggf. auf unterschiedliche Lehrkonzepte zurückzuführen sind. Um dies beantworten zu können, wurden jene ProbandInnen ausgewählt, die Deutsch als L1 sprechen und die Russisch (und auch Polnisch) nicht im natürlichen Kontext erworben haben. Zudem sollten sie sich nicht länger in einem russischsprachigen Land aufgehalten haben und Russisch nur (bzw. überwiegend) im Unterricht verwenden, sodass von prototypischen FremdsprachenlernerInnen gesprochen werden kann, die ihre Sprachkompetenzen vorwiegend aus dem fremdsprachlichen Unterricht (und logischerweise dessen Aufbereitung) beziehen. Diesen Kriterien entsprachen insgesamt 18 Personen: drei aus der Universität, sieben aus dem Weiterbildungskolleg und acht aus dem Gymnasium. Von ihnen wurde aus den erhobenen Tonaufnahmen das Vorlesen eines kurzen Textes auf A1-Niveau analysiert. Dieser Text umfasste 77 Wortformen und wurde ohne Betonungszeichen präsentiert. Die Aufgabe lautete, ihn ohne vorheriges Durchlesen laut vorzulesen. In die Analyse flossen die folgenden qualitativen und quantitativen Kriterien ein:

- Vorlesegeschwindigkeit (Umrechnung in Wörter pro Sekunde);

- Aussprache von <-ого> ([ovə/əva] und mögliche andere Varianten ohne Akanje bzw. [ogo] mit ebenfalls anderen möglichen Varianten mit Akanje);

- Grundsätzliches Verständnis des Betonungssystems ([wechselnde] Betonung eines Vokales im Wort und in der Folge die anderen Vokale unbetont und reduziert);

- Anzahl der korrekten Betonungen und des resultierenden Akanje;

- Aussprache von ausgewählten frequenten Wörtern aus dem Text (diese sind: ona, govorit, tol'ko, plocho und po). 
Die Ergebnisse (s. Tab. 1) sollen quantitativ dargestellt werden, es sei jedoch darauf hingewiesen, dass es sich um jeweils kleine ProbandInnenzahlen handelt und in vielen Fällen eine qualitative Auswertung dahintersteht. Damit sind die angegebenen Zahlen als Orientierung einzuschätzen, die keiner Signifikanzprüfung unterzogen wurden (diese sehe ich im gegebenen Fall ohne interpretativen Mehrwert).

Tabelle 1: Werte zur Vorlesegeschwindigkeit

\begin{tabular}{|l|c|c|c|c|}
\hline \multicolumn{1}{|c|}{ Lernort } & $\begin{array}{c}\text { Wörter/Sek.: } \\
\text { Mittelwert }\end{array}$ & $\begin{array}{c}\text { Standard- } \\
\text { abweichung }\end{array}$ & Median & $\begin{array}{c}\text { Höchster Wert } \\
\text { (Wörter/Sek.) }\end{array}$ \\
\hline Universität & 1,33 & 0,65 & 1,04 & 2,08 \\
\hline $\begin{array}{l}\text { Weiterbildungs- } \\
\text { kolleg }\end{array}$ & 0,81 & 0,15 & 0,82 & 1,05 \\
\hline Gymnasium & 0,85 & 0,26 & 0,77 & 1,43 \\
\hline
\end{tabular}

Wie aus den Zahlen ersichtlich, schneidet die universitäre Gruppe in Bezug auf die Vorlesegeschwindigkeit am besten ab. Mit dem höchsten Wert von 2,08 Wörtern pro Sekunde (W/S) ist bei einem Probanden eine normale Sprechgeschwindigkeit gegeben, der langsamste Sprecher liegt mit 0,88 W/S immer noch über dem Mittelwert der anderen beiden Gruppen. Zugleich ist hier jedoch die Spannbreite am höchsten, was auch durch die sehr kleine ProbandInnenzahl begründbar ist. Zwischen dem Weiterbildungskolleg und dem Gymnasium lässt sich aus dem Mittelwert zunächst kein nennenswerter Unterschied ablesen - die LernerInnen aus dem Gymnasium scheinen hier im Mittel geringfügig schneller zu sein. Bezieht man jedoch die Standardabweichung und den Median mit in die Betrachtung ein, so kann festgestellt werden, dass sich die Gruppe aus dem Weiterbildungskolleg insgesamt homogener in der Vorlesegeschwindigkeit verhält und sich - auf die Gruppe verteilt - eine gleichmäßigere Sprechgeschwindigkeit ermitteln lässt, während für das Gymnasium der höhere Mittelwert maßgeblich auf einen ,Ausreißer' mit einer Zeit von 1,43 W/S zurückzuführen und der Rest der Gruppe unter dem Wert des Weiterbildungskollegs zu verorten ist. 
Dieser Eindruck (Universität vor Weiterbildungskolleg und Gymnasium, die etwa gleich auf sind, bei leicht besserem Abschneiden des Weiterbildungskollegs) bestätigt sich noch stärker unter Berücksichtigung weiterer Kriterien. In die Auswertung der Aussprache von <-oro $>$ floss der Wechsel von $<\mathrm{g}>\mathrm{zu}[\mathrm{v}]$ ein, Besonderheiten der Betonung wurden hier nicht berücksichtigt. Diese wurden in drei unterschiedlichen Schritten analysiert: Von einem grundsätzlichen Verständnis des russischen Betonungssystems wurde dann ausgegangen, wenn aus der Aussprache klar wurde, dass eine Silbe die Betonung trägt (und daher der Vokal in der Silbe betont ausgesprochen wird), während die anderen unbetont sind und die dort enthaltenen Vokale in der Folge reduziert werden. Dies setzt nicht voraus, dass die Betonung auf der korrekten Silbe erfolgt; so werden Fälle wie [p'ırvovə] ebenso gezählt wie das korrekte [p’ervəvə] (der betonte Vokal ist unterstrichen). Im zweiten Schritt wurde die Anzahl aller korrekt betonten (und reduzierten) Wörter ausgezählt und im dritten Schritt die korrekte Betonung von fünf im Russischen frequent vorkommenden (und dadurch auch im Fremdsprachenunterricht vermuteten häufigen) Wörtern betrachtet. Die Ergebnisse werden in Abb. 1 dargestellt (Angaben jeweils in \%):

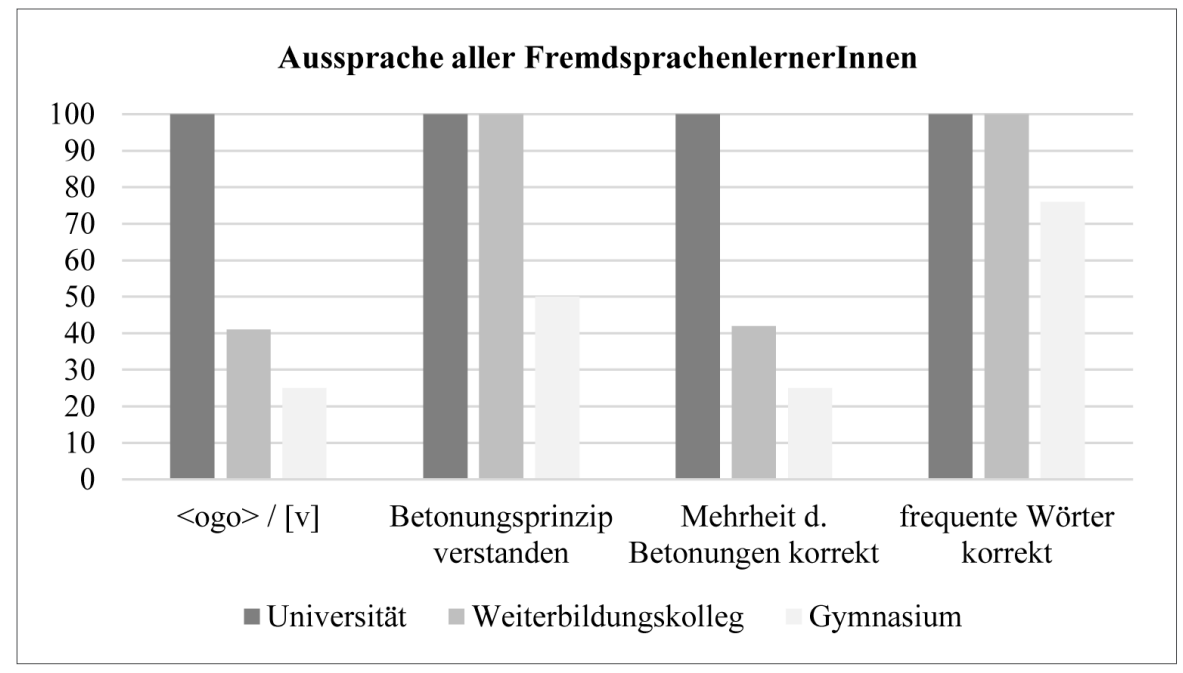

Abbildung 1: Auswertung der Aussprache aller FremdsprachenlernerInnen, Häufigkeiten in \% 
Abb. 1 zeigt, dass die universitäre Gruppe in allen Bereichen die deutlich höchste Performanz bei allen ProbandInnen zeigt. Das Weiterbildungskolleg positioniert sich in der Mitte, wobei auch hier wiederum ein recht homogenes Ausspracheverhalten auffällt: Alle ProbandInnen haben das grundsätzliche Prinzip verstanden und können dieses auch im Fall von häufigen Wörtern anwenden. Die korrekte Betonung von anderen Wörtern verteilt sich auf unterschiedliche ProbandInnen, während bei der Frage von $<$ ogo $>$ zu [v] einzelne dieses Prinzip korrekt angewendet haben, während es andere nie berücksichtigen. In der gymnasialen Gruppe hingegen fallen alle Ergebnisse am schlechtesten aus. Zusätzlich ist die korrekte Aussprache in allen Bereichen vorrangig einer Probandin zuzuordnen (jener mit der schnellsten Vorlesegeschwindigkeit). Damit zeigen sich doch recht deutliche Unterschiede zwischen den drei Lernorten. ${ }^{8}$

Versucht man nun, diese aufgefundenen Unterschiede zu begründen, so lassen sich einige Ansätze finden, die wohl in der Summe ein Gesamtbild zeichnen können. Für die Universität ist vorrangig eben jener universitäre Hintergrund relevant, aus dem sowohl ein höheres Alter hervorgeht als auch eine andere Motivation und ein anderes Interesse, die Sprache zu lernen (freigewähltes Studienfach mit wahrscheinlich einhergehendem Sprachinteresse und Lernneigung, in allen Fällen weisen die ProbandInnen Kenntnisse in mehr als einer weiteren Fremdsprache auf). Somit unterscheidet sich diese Gruppe von den anderen beiden in sehr relevanten Punkten. Dies erklärt jedoch nicht die abweichenden Performanzen aus Gymnasium und Weiterbildungskolleg, die zwar im Alter einen geringfügigen Unterschied aufweisen, ansonsten aber sehr gut vergleichbare Hintergründe bieten (v. a. FremdsprachenlernerInnen der zweiten Fremdsprache an einer Schule mit grundsätzlich freier Entscheidung für Russisch). Als maßgeblicher Unterschied fallen zwei Faktoren auf: Zum einen der Unterschied in der Kontaktzeit mit dem Russischen; während die SchülerInnen aus dem Weiterbildungskolleg seit der ersten Klasse Russischunterricht haben und damit schon seit mehreren Jahren regelmäßig mit dem Russischen in Kontakt stehen, trifft dies für die Lernergruppe aus dem Gymnasium erst seit zwei bis drei Jahren zu.

8 Unter Rückgriff auf die Ergebnisse von Bobb (2016, s. o.) lässt sich damit festhalten, dass die Gruppen aus dem Gymnasium in den Bereichen Grammatik und Wortbildung besser abschneiden als jene aus dem Weiterbildungskolleg, letztere sich hingegen im phonetischen Bereich stärker präsentieren.

Anka Bergmann, Olga Caspers \& Wolfgang Stadler (Hg.)

Didaktik der slawischen Sprachen - Beiträge zum 1. Arbeitskreis in Berlin (12.-14.9.2016)

(C) 2018 innsbruck university press, ISBN 978-3-903187-11-5, DOI 10.15203/3187-11-5 
Zum anderen lässt sich die unterschiedliche didaktische Ausgestaltung des Unterrichts anführen; im Weiterbildungskolleg lag lange Zeit der Schwerpunkt auf rhythmischen Elementen und eigenem Sprechen (z. B. auch durch Wiederholen und Einüben von Liedern, Gedichten etc.), was sich zwangsläufig auf die Phonetik auswirkt, während aus den Interviews mit Lehrkräften aus dem Gymnasium deutlich wird, dass, speziell in den Teilen des Unterrichts, in denen es um das laute Vorlesen von Texten geht, die Lehrkräfte auf die HerkunftssprecherInnen im Sinne eines guten Vorbildes für alle zurückgreifen. Dies mag zwar für die HerkunftssprecherInnen von Vorteil sein (auch im Sinne einer Kompetenzbestätigung für diese), führt aber nicht dazu, dass die FremdsprachenlernerInnen ihre Lesekompetenz und Aussprache trainieren können - reines Zuhören genügt hier ganz offensichtlich nicht.

\section{Zusammenfassung und Ausblick}

Die vorliegenden Ausführungen sollen einen Einblick in das Studienprojekt zum Stand des RAF im Ruhrgebiet ermöglichen. Dabei war es das Ziel, zunächst einen Überblick über die Datensammlung, die befragten Personen, Lernorte und didaktischen Konzepte zu präsentieren. Mit der Darstellung einiger zentraler soziolinguistischer Hintergründe der ProbandInnen (hinsichtlich Sprachbiographie, Lernmotivation etc.) konnte ein zwar kleiner, aber konkreter Ausschnitt aus der Lage des Russischunterrichts in Deutschland im Allgemeinen (so auch hinsichtlich der zahlenmäßigen Verteilung von HerkunftssprecherInnen und FremdsprachenlernerInnen und den Möglichkeiten, an unterschiedlichen Einrichtungen Russisch zu erwerben) beschrieben werden. Als eindrückliches Ergebnis sei hier auf die Angabe des Motivationsgrades und der Gründe, Russisch zu lernen und die Unterschiede zwischen HerkunftssprecherInnen und FremdsprachenlernerInnen hingewiesen: Letztere sind grundsätzlich motivierter und haben sehr gut überlegte Gründe, Russisch zu lernen, während dies für die HerkunftssprecherInnen in nicht so ausgeprägtem Maße zutrifft. Dies geht einher mit einem geringeren Spaß am Russischunterricht. Dieses Ergebnis lässt großen Raum für zukünftige Überlegungen zur Ausgestaltung des Russischunterrichts hinsichtlich einer ande-

Anka Bergmann, Olga Caspers \& Wolfgang Stadler (Hg.)

Didaktik der slawischen Sprachen - Beiträge zum 1. Arbeitskreis in Berlin (12.-14.9.2016)

(C) 2018 innsbruck university press, ISBN 978-3-903187-11-5, DOI 10.15203/3187-11-5 
ren Einbindung und Förderung von HerkunftssprecherInnen auch in den Einrichtungen, in denen die TeilnehmerInnenzahlen für rein herkunftssprachliche Gruppen nicht ausreichen. Dass nämlich nicht zuletzt die Motivation und der Spaß am Lernen der Strukturen der Sprache ausschlaggebend sind für den Erwerb von Sprachkenntnissen, ist ein weiteres Ergebnis dieses Projekts (s. Kandt 2016). Der konstruktive Umgang mit sprachlicher Heterogenität ist damit nach wie vor eine der zentralen Aufgaben des Russischunterrichts als Fremdsprache.

Mit der exemplarischen Darstellung von ersten Ergebnissen sollte die Breite des gesammelten Materials und die Reichweite der daraus resultierenden Fragestellungen präsentiert werden. Dafür wurden bewusst sehr unterschiedliche Blickwinkel eingenommen, um möglichst viele InteraktantInnen des Russischunterrichts zu berücksichtigen: Sei es die immer wieder diskutierte Frage nach den Möglichkeiten und Hinlänglichkeiten der Leistungsbeurteilung und die gute Übereinstimmung der unterschiedlichen Methoden, die Beleuchtung der Rolle der Lernermotivation als wichtigen lernerseitigen Faktor, die Beschreibung des aktuellen Standes eines Ausschnitts aus dem Lernerlexikon mithilfe von Wortassoziationen (systemlinguistische Perspektive) oder der Einblick in die Auswirkung unterschiedlicher didaktischer Konzepte bzw. institutioneller Rahmenbedingungen auf die Performanz in einem konkreten Bereich (Vorleseperformanz). Zwar sind die hier dargestellten Ergebnisse bislang an jeweils geringen ProbandInnenzahlen gewonnen, dennoch haben sie eine Aussagekraft in dem Sinne, dass es sich lohnt, diesen (und weiteren) Fragestellungen auch auf Basis der vorgestellten Methoden vertieft nachzugehen, diese in weiteren Studien auszubauen und so zu umfassenderen Ergebnissen der unterschiedlichen Faktoren zu gelangen, die den Russischunterricht ausmachen und somit zum Lernerfolg in den dort häufig aufzufindenden heterogenen Gruppen beizusteuern.

\section{Literaturverzeichnis}

Anstatt, T. (2008). Wer ,dunkel' hört, muss nicht ,hell' sagen: Wortassoziationen in slavischen und germanischen Sprachen. In P. Kosta \& D. Weiss (Hrsg.), Slavistische Linguistik 2006/2007. Referate des XXXII. und XXXIII. Konstanzer Slavistischen Arbeitstreffens (11-34). München: Sagner.

Anka Bergmann, Olga Caspers \& Wolfgang Stadler (Hg.)

Didaktik der slawischen Sprachen - Beiträge zum 1. Arbeitskreis in Berlin (12.-14.9.2016)

(C) 2018 innsbruck university press, ISBN 978-3-903187-11-5, DOI 10.15203/3187-11-5 
Bergmann, A. (Hrsg.) (2014). Fachdidaktik Russisch: Eine Einführung. Tübingen: Narr.

Bobb, A. (2016). Sprachstand von Russischlernern in Fremd-und Selbsteinschätzung: Empirische Untersuchung. Unveröffentlichte Hauptseminarhausarbeit, Ruhr-Universität Bochum. Bochum.

Brown, R. \& Berko, J. (1960). Word Association and the Acquisition of Grammar. Child Development, 31 (1), 1-14.

Hartenstein, K. (2000). Zur Rolle von explizitem und implizitem Wissen beim instruktionsgeleiteten L2-Grammatikerwerb - eine empirische Untersuchung am Beispiel der Lang- und Kurzformen der russischen Adjektive. In C. Riemer (Hrsg.), Kognitive Aspekte des Lehrens und Lernens von Fremdsprachen. Festschrift für Willis J. Edmondson (263-280). Tübingen: Narr.

Henning, G. H. (1973). Remembering Foreign Language Vocabulary: Acoustic and Semantic Parameters. Language Learning - A Journal of Research in Language Studies, 23 (2), 185-196.

Kandt, C. (2016). Der Einfluss des kognitiven Faktors der Sprachbewusstheit auf die Motivation. Unveröffentlichte Hauptseminarhausarbeit, Ruhr-Universität Bochum. Bochum.

Peschel, J., Senger, U. \& Willige, J. (2006). Fremdsprachenkenntnisse - Subjektive Einschätzung und objektiver Test. (HISBUS-Kurzbericht 12). Hannover: HIS.

Raupach, M. (2002). „Explizit/implizit“ in psycholinguistischen Beschreibungen - eine unendliche Geschichte. In W. Börner \& K. Vogel (Hrsg.), Grammatik und Fremdsprachenerwerb. Kognitive, psycholinguistische und erwerbstheoretische Perspektiven (101-117). Tübingen: Narr.

Röhr-Sendlmeier, U. M. (Hrsg.) (2012). Inzidentelles Lernen. Wie wir beiläufig Wissen erwerben. Berlin: Logos.

Schlotthauer, A. (2016). Wortassoziationen bei Fremdsprachenlernern des Russischen. Unveröffentlichte Bachelorarbeit, Ruhr-Universität Bochum. Bochum.

Stadler, W. (2014). Evaluation im Russischunterricht - Testformate Linguistische Kompetenz: Grammatik und Lexik oder Sprache im Kontext testen. In A. Bergmann (Hrsg.), Fachdidaktik Russisch: Eine Einführung (279-312). Tübingen: Narr.

Targońska, J. (2012). Wortbildungsübungen aus der Sicht der Fremdsprachendidaktik und der DaF-Lernenden. Linguistica Silesiana, 33, 63-79.

Trušina, L. B. (1974). Slovoobrazovanie v sisteme prepodavanija russkogo jazyka inostrancam. Russkij jazyk za rubežom, 2, 69-72. 